\title{
A STUDY OF FINITE VOLUME METHODS FOR VISCOELASTIC FLOWS
}

\section{P. WAPPEROM, M.F. WEBSTER}

Institute of Non-Newtonian Fluid Mechanics, Department of Computer Science University of Wales, Swansea, Singleton Park, Swansea SA2 8PP, U.K.

\section{INTRODUCTION}

This study investigates the application of a new hybrid finite element/finite volume scheme to the numerical solution of some viscoelastic flows. Such an FE/FV approach (see similarly Sato and Richardson [3]) is contrasted against a purely finite element alternative, that has previously been developed to address highly elastic problems, see Matallah et al. [2]. For incompressible viscoelastic flows, with non-trivial source terms, the question arises as to whether a finite element approach may be best suited to solve for the parabolic field equations, concerned with the conservation of mass and momentum, whilst a finite volume approach may be more appropriate for the hyperbolic constitutive law, as originally devised. For example, recent work on advection equations has shown that fluctuation distribution schemes that handle upwinding for fluxes, can yield accurate solutions for problems that manifest steep gradients, see Struijs et al. [4]. Efficiency, accuracy and stability are the prime considerations, to be achieved through a localised control volume view, high resolution TVD-type schemes and oscillation-free transient solutions. Computational tractability follows as a consequence.

\section{THEORY}

The basic equations are those derived from conservation of mass and momentum, and transport of stress. The finite element framework, around which this work is centred and compared to, is a semi-implicit second-order time-stepping Taylor-Galerkin/Pressure-Correction scheme of three fractional stages, that incorporates consistent Petrov-Galerkin streamline upwinding and recovery in the discrete treatment of the constitutive equation (FE/SUPG). In two dimensions, the finite element grid is constructed as a triangular tessellation, with pressure nodes located at the vertices (linear representation) and velocity/stress components at both vertices and mid- side nodes (quadratic representation). In the FE/FV hybrid scheme, a cell vertex approach (Crumpton et al. [1]) is adopted in the FV part for the constitutive equation. Lax-Wendroff time-stepping is built into this scheme, as with the FE scheme above. Four linear FV triangular cells are constructed as subcells of each parent quadratic FE triangular cell, connecting the mid-side nodes. With stress variables located at the vertices of the FV cells, no interpolation is required to recover the $\mathrm{FE}$ nodal stress values. The governing FV stress matrix is the identity matrix. For the FE method it is sparse, and necessitates the solution of a matrix-vector equation, hereby with an iterative method as for the momentum equation. This step is circumvented using the hybrid FE/FV, with straightforward right-hand side construction, proving a particular advantage for three-dimensional or multimode computations.

Various different alternative treatments are considered for dealing with flux and source terms, capturing such properties as positivity and linearity preservation. This introduces the concept of both linear (LDB and N-scheme) and non-linear (PSI-scheme) fluctuation distribution schemes [4]. Our objective is to compare and contrast the discrete implementation of these various schemes, to indicate their relative strengths and weaknesses. As their initial development was for pure convection problems, here their extension is considered also for those with source terms, as for an Oldroyd-B benchmark flow.

\section{PROBLEM DESCRIPTION}

Orders of accuracy and efficiency in attaining solutions are established for a two-dimensional Cartesian test problem with analytical solutions on a unit square domain, $\left(x_{0}, x_{1}\right) \mathrm{x}\left(y_{0}, y_{1}\right)$ with $x_{0}=1, x_{1}=2$. This problem may be stated in pure convection form, or for an Oldroyd-B model that exhibits source terms. Structured, 
uniform, quadrilateral-based, triangular finite element meshes are used to test for accuracy, of $2 \times 2,4 \times 4,8 \times 8$ and $16 \times 16$ elements. For the velocity field, we define $u_{x}=x$ and $u_{y}=-y$. Boundary conditions for individual components must be specified, for stress these are required on the inflow boundaries $x=x_{0}$ and $y=y_{1}$.

\section{RESULTS}

For the pure convection problem and comparison of $\mathrm{FE}$ versus flux distribution schemes, the difference from the exact solution with mesh refinement, a fixed velocity field, linear representation of stress, and domain $1\left(y_{0}=1, y_{1}=2\right)$, are displayed in Table 1 . Comparison is made

Table 1: Error norm behaviour for pure convection.

\begin{tabular}{c|c|c|c|c}
\hline & FE & \multicolumn{3}{|c}{ FV } \\
\hline & SUPG & N & LDB & PSI \\
\hline 2 & $2.010^{-3}$ & $1.910^{-2}$ & $1.910^{-3}$ & $2.410^{-3}$ \\
4 & $2.810^{-4}$ & $1.210^{-2}$ & $4.810^{-4}$ & $5.210^{-4}$ \\
8 & $3.810^{-5}$ & $6.410^{-3}$ & $1.210^{-4}$ & $1.410^{-4}$ \\
16 & $5.010^{-6}$ & $3.410^{-3}$ & $1.210^{-4}$ & $3.510^{-5}$ \\
\hline
\end{tabular}

on maximum error norm behaviour throughout, here between the FE/SUPG, N-scheme (linear, positive), LDB-scheme (linear, linearity preserving) and PSI-scheme (nonlinear, linearity preserving, positive). The linearised advection velocity approach, is assumed initially and results are characterised via component scalar solutions of the form $1+(x y)^{0.5}$, see Wapperom and Webster [5]. The FE data shows almost $\mathcal{O}\left(h^{3}\right)$ convergence. What is immediately clear for this model problem, is that the linearity preservation property is essential to obtain the higher levels of accuracy. The PSI- and LDBscheme display $\mathcal{O}\left(h^{2}\right)$ convergence, whilst the $\mathrm{N}$-scheme only achieves $\mathcal{O}\left(h^{0.8}\right)$. Furthermore, the LDB-scheme has a wider stability range on time step, practically double that of the others. The LDB-scheme renders the fastest convergence rate, that results in $2 \%$ of the $\mathrm{FE} \mathrm{CPU}$ time.

For the Oldroyd-B flow problem that has source terms, we compare in Table 2 the FV method employing linear (FVL) and quadratic integral (FVQ) evaluation with FE/SUPG for the more severe test case of domain $2\left(y_{0}=0.1\right.$, $\left.y_{1}=1.1\right)$, where the velocity is almost parallel to the lower boundary. For this problem and domain 1, FVL- and FVQ-schemes are approximately second-order accurate and FE/SUPG is almost cubic. On domain 2, FE/SUPG achieves

Table 2: Error norm behaviour of $\tau_{x x}$ for Oldroyd-B.

\begin{tabular}{c|c|c|c}
\hline & FE/SUPG & FVL & FVQ \\
\hline 2 & $1.410^{-3}$ & $2.210^{-3}$ & $7.310^{-4}$ \\
4 & $3.610^{-4}$ & $1.210^{-3}$ & $1.910^{-4}$ \\
8 & $6.510^{-5}$ & $5.410^{-4}$ & $3.610^{-5}$ \\
16 & $9.710^{-6}$ & $2.310^{-4}$ & $5.710^{-6}$ \\
\hline
\end{tabular}

$\mathcal{O}\left(h^{2.3}\right)$ accuracy. Table 2 shows the loss of accuracy with linear integral evaluation; this appears in all components. In worst case, we observe $\mathcal{O}(h)$ convergence for $\tau_{x x}$. In contrast, we recover the desired $\mathcal{O}\left(h^{2}\right)$ convergence in FVQ with quadratic integral evaluation.

\section{CONCLUSIONS}

The introduction of source terms in a consistent manner, does not detract from the accuracy of the FV scheme. The effective coupling of the FE and FV components of the hybrid scheme has been demonstrated elsewhere [5], with accuracy between $\mathcal{O}\left(h^{2}\right)$ and $\mathcal{O}\left(h^{3}\right)$, hence showing no deterioration from the FE discretisation. As the FVQ implementation for stress alone takes $10 \%$ of the time for its FE counterpart, solution time is reduced to that of the base Navier-Stokes solver. Hence, a considerable gain in efficiency is anticipated with this hybrid $\mathrm{FE} / \mathrm{FV}$ scheme for large problems, involving either multi-mode or three-dimensional calculations.

\section{References}

[1] Crumpton PI, Mackenzie JA, Morton KW (1993) J Comput Phys 109: 1-15

[2] Matallah H, Townsend P, Webster MF (1998) J Non-Newtonian Fluid Mech 75: 139-166

[3] Sato T, Richardson SM (1994) J NonNewtonian Fluid Mech 51: 249-275

[4] Struijs R, Deconinck H, Roe PL (1991) Lecture series 1990-01, Von Karman Institute for Fluid Dynamics

[5] Wapperom P, Webster MF in press J NonNewtonian Fluid Mech 